\title{
POLYNOMIAL ENDOMORPHISMS PRESERVING OUTER RANK IN TWO VARIABLES
}

\author{
YONG JIN and XIANKUN DU ${ }^{凶}$
}

(Received 22 September 2011)

\begin{abstract}
An endomorphism $\varphi$ of a polynomial ring is said to preserve outer rank if $\varphi$ sends each polynomial to one with the same outer rank. For the polynomial ring in two variables over a field of characteristic 0 we prove that an endomorphism $\varphi$ preserving outer rank is an automorphism if one of the following conditions holds: (1) the Jacobian of $\varphi$ is a nonzero constant; (2) the image of $\varphi$ contains a coordinate; (3) $\varphi$ has a 'fixed point'.
\end{abstract}

2010 Mathematics subject classification: primary 14R10.

Keywords and phrases: outer rank, coordinate, test polynomial, retract.

\section{Introduction}

The outer rank of an element has been considered for several algebraic systems, for example for free groups in [1, 10, 15] and for free Lie algebras in [12]. However, to our knowledge no systematical work on outer rank has been done for polynomial rings.

The outer rank of a polynomial $p$, abbreviated as $\operatorname{Orank}(p)$, is the minimum number of variables on which an automorphic image of $p$ can depend. It is easy to see that the outer rank of an element is invariant under automorphisms. Conversely, it is natural to ask whether an endomorphism preserving outer rank is an automorphism. We say that an endomorphism $\varphi$ of a polynomial ring preserves outer rank (the outer rank $i$, respectively) if $\varphi$ sends each polynomial (of outer rank $i$, respectively) to one with the same outer rank. This problem was first posed in [16], where it is called outer rank preserving problem, and the author showed that the answer is positive for an endomorphism with a nonzero constant Jacobian in the case of algebraically closed fields. Similar preserving problems have been considered by several authors, for example, the coordinate preserving problem in $[5,6,8]$ and the automorphic orbit preserving problem in $[7,17]$.

Supported by NSF of China (No.11071097) and '211 Project' and '985 Project' of Jilin University.

(C) 2012 Australian Mathematical Publishing Association Inc. 0004-9727/2012 \$16.00 
Throughout this paper $k[X]:=k\left[x_{1}, x_{2}, \ldots, x_{n}\right]$ denotes the polynomial ring in the variables $x_{1}, x_{2}, \ldots, x_{n}$ over a field $k$ of characteristic 0 , and we write $k[x, y]$ instead of $k\left[x_{1}, x_{2}\right]$ if $n=2$. Let $\operatorname{End}(k[X])$ and $\operatorname{Aut}(k[X])$ denote the set of endomorphisms and the group of automorphisms of $k[X]$, respectively. This paper focuses on $k[x, y]$.

Shpilrain and Jie-tai Yu [13] proved that an endomorphism $\varphi$ of $k[x, y]$ with the Jacobian being a nonzero constant is an automorphism if one of the following conditions holds.

(1) The image of $\varphi$ contains a coordinate.

(2) $\varphi$ has a 'fixed point'.

(3) $\varphi^{\infty}(k[x, y]) \neq k$, where $\varphi^{\infty}(k[x, y])=\bigcap_{i=1}^{\infty} \varphi^{i}(k[x, y])$.

The aim of this paper is to prove that analogous results hold when replacing the Jacobian condition by preserving outer rank, which gives partial solutions to the outer rank preserving problem. It is also interesting to compare the results of [17] with those of the present paper. In [17] the author assumes that $\varphi$ preserves automorphic orbit, a stronger assumption, but only on one element, while we assume that $\varphi$ preserves outer rank, a weaker assumption, but on all elements.

\section{Main results}

We start with a simple observation.

Proposition 2.1. Let $\varphi \in \operatorname{End}(k[X])$. If $\varphi$ preserves outer rank, then $\varphi$ is a monomorphism.

Proof. Suppose that $\varphi$ is not a monomorphism. Then there exists $H(X) \in k[X] \backslash k$ such that $\varphi(H(X))=0$. Since $\varphi$ preserves outer rank, Orank $(H(X))=0$, a contradiction.

Remark 2.2. The converse of Proposition 2.1 is not true; for example, $\varphi=\left(x, y^{2}\right)$ is a monomorphism that does not preserve outer rank one. But we do not know yet if a monomorphism preserving outer rank one is an automorphism.

A subalgebra $A$ of $k[X]$ is called a retract if it is the image of an idempotent (called a retraction) of $\operatorname{End}(k[X])$. A retract different from $k$ and $k[X]$ is called proper. It is easy to see that if $p(x, y)$ generates a proper retract of $k[x, y]$ with a retraction $\varphi$, then $\alpha(p(x, y))$ also generates a proper retract with retraction $\alpha \varphi \alpha^{-1}$ for any $\alpha \in$ $\operatorname{Aut}(k[x, y])$. Retracts have been studied by many authors, among them $[3,7,13,14]$.

The following lemma is a restatement of [13, Theorem 1.1].

Lemma 2.3. A polynomial $p(x, y)$ generates a proper retract of $k[x, y]$ if and only if there exist a $\alpha \in \operatorname{Aut}(k[x, y])$ and $q(x, y) \in k[x, y]$ such that $p(x, y)=\alpha(x+y q(x, y))$.

The following two simple observations will be useful in this paper.

Lemma 2.4. For any $g(X) \in k[X]$ and for any $f \in k[T] \backslash k, g(X)$ and $f(g(X))$ have the same outer rank. 
Proof. The result follows directly from the fact that the numbers of indeterminates on which $\alpha(f(g(X)))$ and $\alpha(g(X))$ respectively depend are the same for any $\alpha \in \operatorname{Aut}(k[X])$.

Lemma 2.5. Let $p(X) \in k[X]$. Then $\operatorname{Orank}(p(X))=1$ if and only if there exist $u(T) \in$ $k[T] \backslash k$ and a coordinate $q(X)$ such that $p(X)=u(q(X))$.

Proof. This follows directly by the definition of outer rank.

Lemma 2.6. Let $p(X), q(X) \in k[X]$ and let $p(X)=u(q(X))$ for some $u(T) \in k[T] \backslash k$. Then $p(X)$ is a coordinate if and only if $\operatorname{deg}(u(T))=1$ and $q(X)$ is a coordinate.

Proof. Suppose that $p(X)$ is a coordinate. Then $\operatorname{Orank}(p(X))=1$ and hence, by Lemma 2.4, Orank $(q(X))=1$. By Lemma 2.5, $q(X)=v(r(X))$ for some coordinate $r(X)$ and $v(T) \in k[T] \backslash k$. Thus $p(X)=(u \circ v)(r(X))$. Since $\operatorname{Orank}(p(X))=1, x_{1}=\alpha(p(X))$ for some $\alpha \in \operatorname{Aut}(k[X])$. Hence $x_{1}=\alpha(p(X))=\alpha((u \circ v)(r(X)))=(u \circ v)(\alpha(r(X)))$, which implies that $\operatorname{deg}(u(T))=\operatorname{deg}(v(T))=1$. It follows that $q(X)$ is a coordinate. The sufficiency is obvious.

Corollary 2.7. Let $\varphi \in \operatorname{End}(k[X])$ preserve outer rank. If $\varphi(p(X))$ is a coordinate, then $p(X)$ is a coordinate.

Proof. Since $\varphi$ preserves outer $\operatorname{rank}, \operatorname{Orank}(p(X))=1$. Hence $p(X)=\alpha\left(u\left(x_{1}\right)\right)$ for some $\alpha \in \operatorname{Aut}(k[X])$ and $u(T) \in k[t] \backslash k$. It suffices to show that $u(T)$ is a coordinate. Since $u\left(\varphi\left(\alpha\left(x_{1}\right)\right)\right)=\varphi(p), \operatorname{deg}(u(T))=1$ by Lemma 2.6. Thus $u(T)$ is a coordinate, as desired.

Remark 2.8. Corollary 2.7 extends the main theorem in [2] to the case of $n$ variables under a more restrictive condition.

The following result is of independent interest.

Lemma 2.9. Suppose that $g(x, y)=x+y q(x, y)$. Then $\operatorname{Orank}(g(x, y))=1$ if and only if $q(x, y) \in k[y]$. Equivalently, $\operatorname{Orank}(g(x, y))=2$ if and only if $q(x, y) \in k[x, y] \backslash k[y]$.

PRoof. It is enough to prove the first conclusion.

Suppose that $q(x, y) \in k[y]$. Then $g(x, y)=x+f(y)$ for some $f(T) \in k[T]$. In this case $g(x, y)$ is a coordinate, and so it is of outer rank one.

Suppose that $\operatorname{Orank}(g(x, y))=1$. Then there exists $\alpha \in \operatorname{Aut}(k[x, y])$ such that $\alpha(g)=$ $f(x)$, and $f(x)$ generates a proper retract by Lemma 2.3. By [13, Corollary 1.2] there is $\varphi \in \operatorname{End}(k[x, y])$ such that $\varphi(f(x))=x$, that is, $f(\varphi(x))=\varphi(f(x))=x$. It follows from Lemma 2.6 that $\operatorname{deg}(f(x))=1$. In particular, $f(x)$ is a coordinate, which implies that $g(x, y)$ is also a coordinate. By [11, Corollary 14] the Newton polygon of $g(x, y)$ is a triangle with edges on the axes, and so by the form of $g(x, y)$ we get $q(x, y) \in k[y]$.

A polynomial $p(X) \in k[X]$ is called a test polynomial if any $\varphi \in \operatorname{End}(k[X])$ with $\varphi(p(X))=p(X)$ is an automorphism. It is shown in [5] that test polynomials are all of maximal outer rank. Polynomials that are of maximal outer rank but are not 
test polynomials were given in [5, 9]; most of these are polynomials in $k[x, y]$. The following corollary shows that such polynomials have a 'standard' form.

Corollary 2.10. Let $h(x, y) \in k[x, y]$. Then $h(x, y)$ is of outer rank two and is not a test polynomial if and only if there exist $q(x, y) \in k[x, y] \backslash k[y]$ and an automorphism $\alpha$ such that $h(x, y) \in k[\alpha(x+y q(x, y))]$.

Proof. By [7, Theorem 1.1] $h(x, y)$ is not a test polynomial if and only if $h(x, y)$ is contained in a proper retract. By [3, Theorem 3.5] every proper retract is generated by a polynomial $g(x, y) \in k[x, y]$. Furthermore, by Lemma 2.3, $g=\alpha(x+y q(x, y))$ for some $\alpha \in \operatorname{Aut}(k[x, y])$ and $q(x, y) \in k[x, y]$. Note that automorphisms preserve outer rank. Orank $(h(x, y))=2$ if and only if $\operatorname{Orank}(x+y q(x, y))=2$ by Lemma 2.4, if and only if $q(x, y) \in k[x, y] \backslash k[y]$ by Lemma 2.9, which completes the proof.

REMARK 2.11. Examples given in [5,9] can easily be figured out by Corollary 2.10.

The following example given in [4] can be proved directly by Corollary 2.10.

Corollary 2.12 [4, Example 3.1]. Let $p(x, y)=x^{2}+y^{2} \in k[x, y]$, where $k$ contains $\sqrt{-1}$. Then $p(x, y)$ is not a test polynomial.

Proof. Take $\alpha=(f(x, y), g(x, y))$, where $f(x, y)=-3 \sqrt{-1} x-y+1, g(x, y)=\sqrt{-1} x+$ $y-1$. Then $\alpha \in \operatorname{Aut}(k[x, y])$. Set $h(T)=T+1, q(x, y)=x+2 y+3$. Then $p(x, y)=$ $h(\alpha(x+y q(x, y)))$. Now Corollary 2.10 implies that $p(x, y)$ is of outer rank two and not a test polynomial.

The following result was first proved in [16] for an arbitrary $n$ and an algebraically closed field $k$. In fact, in the case $n=2$ it also holds for any field of characteristic 0 , and we will give its proof for completeness.

For $\varphi \in \operatorname{End}(k[X])$ we denote by $J_{\varphi}$ the Jacobian of $\varphi$.

Proposition 2.13. Let $\varphi \in \operatorname{End}(k[x, y]$ ), where $k$ is a field of characteristic 0 (not necessarily algebraically closed). If $\varphi$ preserves outer rank one and $J_{\varphi} \in k^{*}$, then $\varphi$ is an automorphism.

Proof. By [5, Theorem 1.1] it is sufficient to show that $\varphi$ sends coordinates to coordinates. Suppose that $p(x, y)$ is a coordinate and $q(x, y)=\varphi(p(x, y))$. Then $q(x, y)$ is of outer rank one. So there are $\alpha, \beta \in \operatorname{Aut}(k[x, y])$ such that $\alpha(p(x, y))=x$ and $\beta(q(x, y))=u(x)$ for some $u(x) \in k[x] \backslash k$. We need to show that $u(x)$ is a coordinate. Set $\psi=\beta \varphi \alpha$. Then $J_{\psi} \in k^{*}$ by the chain rule. Observing that $\psi(x)=u(x)$, we see that $J_{\psi}=\partial_{x}(u(x)) \partial_{y}(\psi(y))$. Thus we get $u(x)=a x+b$ and $\psi(y)=c y+r(x)$ for some $a, b \in k^{*}, c \in k$ and $r(x) \in k[x]$. In particular, $u(x)$ is a coordinate. We are done.

The following example shows that the condition ' $J_{\varphi} \in k^{*}$ ' in Proposition 2.13 cannot be removed.

ExAmple 2.14. Let $\varphi=\left(x^{2}, x^{3}\right) \in \operatorname{End}(k[x, y])$. Then $\varphi$ preserves outer rank one and is not an automorphism. 
Proof. Notice that $\varphi$ is not an automorphism and does not increase outer rank. So to show that $\varphi$ preserves outer rank one, we just need to show that it cannot decrease the outer rank of a polynomial of outer rank one.

Suppose, to the contrary, that there is $p(x, y)$ with outer rank one such that $\operatorname{Orank}(\varphi(p))=0$. Then, by Lemma 2.5, $\operatorname{Orank}(p(x, y))=1$ implies that $p=u(q)$ for some $u(T) \in k[T] \backslash k$ and a coordinate $q(x, y)$, while $\operatorname{Orank}(\varphi(p(x, y)))=0$ implies that $\varphi(p(x, y)) \in k$. It follows that

$$
u(\varphi(q(x, y)))=\varphi(u(q))=\varphi(p(x, y)) \in k,
$$

whence $\varphi(q(x, y)) \in k$. Thus $q\left(x^{2}, x^{3}\right)=q(\varphi(x), \varphi(y))=\varphi(q(x, y)) \in k . \quad$ Because $q(x, y)$ is a coordinate, $q(x, y)=a x+b y+h(x, y)$, where $a, b$ are not both 0 and $h(x, y) \in k[x, y]$ has no terms of degree one. Thus $a x^{2}+b x^{3}+h\left(x^{2}, x^{3}\right)=\varphi(q) \in k$. Equivalently, $a x^{2}+b x^{3}=-h\left(x^{2}, x^{3}\right)+c$ for some $c \in k$. By comparing the degree, we get a contradiction.

It is worth pointing out that Example 2.14 also shows that endomorphisms preserving outer rank one are not necessarily automorphisms.

We are now in a position to prove our main theorem.

THeORem 2.15. Let $\varphi \in \operatorname{End}(k[x, y])$. Suppose that $\varphi$ preserves outer rank. If there is a coordinate in $\varphi(k[x, y])$, then $\varphi$ is an automorphism.

Proof. Suppose that there exists a coordinate $q(x, y)$ such that $q(x, y)=\varphi(p(x, y))$ for some $p(x, y) \in k[x, y]$. Then $\operatorname{Orank}(p(x, y))=1$ by hypothesis, which implies that $p(x, y)=\alpha(v(x))$ for some $\alpha \in \operatorname{Aut}(k[x, y])$ and $v(x) \in k[x] \backslash k$. Thus $q(x, y)=$ $\varphi \alpha(v(x))=v(\varphi \alpha(x))$. Set $\varphi \alpha(x)=f(x, y)$. Then $q(x, y)=v(f(x, y))$, and so $f(x, y)$ is a coordinate by Lemma 2.6. Then there exists $\beta \in \operatorname{Aut}(k[x, y])$ such that $\beta(f(x, y))=x$. It follows that $\beta \varphi \alpha(x)=\beta(f(x, y))=x$. Let $g(x, y)=\beta \varphi \alpha(y)$. By composing $\beta \varphi \alpha$ with $(x, y-g(x, 0)) \in \operatorname{Aut}(k[x, y])$, without loss of generality, we may suppose that $\varphi=(x, y h(x, y))$.

Note that $\varphi(x+y)=x+y h(x, y)$. Since $\operatorname{Orank}(x+y)=1$ and $\varphi$ preserves outer rank, Orank $(x+y h(x, y))=1$. By Lemma 2.9, $h(x, y) \in k[y]$. So $\varphi=(x, g(y))$, where $g(y)=y h(x, y) \in k[y]$. Since $\varphi$ is a monomorphism by Proposition 2.1, $x$ and $g(y)$ are algebraically independent, forcing that $g(y) \neq 0$.

To prove that $\varphi \in \operatorname{Aut}(k[x, y])$, it suffices to show that $g(y)=a y$ for some $a \in k^{*}$. Note that $\varphi\left(x^{2}+y\right)=x^{2}+g(y)$. Since $\operatorname{Orank}\left(x^{2}+y\right)=1$ and $\varphi$ preserves outer rank, $\operatorname{Orank}\left(x^{2}+g(y)\right)=1$, which implies that there exists $\alpha \in \operatorname{Aut}(k[x, y])$ such that $\alpha\left(x^{2}+\right.$ $g(y))=t(x)$, where $t(x) \in k[x] \backslash k$. Hence $\operatorname{grad}(u(x, y))$ and $\operatorname{grad}(t(x))$ have the same number of zeros, where $u(x, y)=x^{2}+g(y)$ and $\operatorname{grad}(u(x, y))$ denotes the gradient of $u(x, y)$. Observe that $\operatorname{grad}(t(x))=\left(\partial_{x}(t(x)), 0\right)$, the number of zeros of which is zero or infinite. On the other hand, $\operatorname{grad}(u)=\left(2 x, \partial_{y}(g(y))\right)$, the number of zeros of which is finite. It follows that $\operatorname{grad}(u)$ has no zero, which implies that $\partial_{y}(g(y))=a \in k^{*}$. Thus $g(y)=a y$, as desired. 
Shpilrain and $\mathrm{Yu}[14$, Proposition] state that if $\varphi$ is a monomorphism of $\mathbb{C}[x, y]$ and there are no coordinates in $\varphi(\mathbb{C}[x, y])$, then $\varphi^{\infty}(\mathbb{C}[x, y])=\mathbb{C}$, where $\varphi^{\infty}(\mathbb{C}[x, y])=$ $\bigcap_{i=1}^{\infty} \varphi^{i}(\mathbb{C}[x, y])$. The authors split the proof into two cases, namely $\operatorname{deg}\left(J_{\varphi}\right)=0$ and $\operatorname{deg}\left(J_{\varphi}\right)>0$. In fact, the proof of the latter case is valid for an arbitrary field $k$ of characteristic 0 . Hence the following proposition holds.

Proposition 2.16. If $\varphi$ is a monomorphism of $k[x, y]$ and there are no coordinates in $\varphi(k[x, y])$, then either $J_{\varphi} \in k^{*}$ or $\varphi^{\infty}(k[x, y])=k$.

The following result is of independent interest.

THeOREM 2.17. Let $\varphi \in \operatorname{End}(k[x, y])$. If $\varphi$ preserves outer rank, then either $\varphi$ is an automorphism or $\varphi^{\infty}(k[x, y])=k$.

Proof. Suppose that $\varphi$ is not an automorphism. Then in view of Proposition 2.1 and Theorem 2.15 we know that $\varphi$ is a monomorphism and there are no coordinates in $\varphi(k[x, y])$. Hence, by Proposition 2.16, either $J_{\varphi} \in k^{*}$ or $\varphi^{\infty}(k[x, y])=k$. But Proposition 2.13 implies that the first case cannot occur. Consequently, we obtain $\varphi^{\infty}(k[x, y])=k$.

Corollary 2.18. Let $\varphi \in \operatorname{End}(k[x, y])$. If $\varphi$ preserves outer rank and $\varphi(u(x, y))=$ $u(x, y)$ for some $u(x, y) \in k[x, y] \backslash k$, then $\varphi$ is an automorphism.

\section{References}

[1] G. M. Bergman, 'Supports of derivations, free factorizations, and ranks of fixed subgroups in free groups', Trans. Amer. Math. Soc. 351 (1999), 1531-1550.

[2] L. A. Campbell and J.-T. Yu, 'Two dimensional coordinate polynomials and dominant maps', Comm. Algebra 28 (2000), 2297-2301.

[3] D. L. Costa, 'Retracts of polynomial algebras', J. Algebra 44 (1977), 492-502.

[4] V. Drensky and J.-T. Yu, 'Test polynomials for automorphisms of polynomial and free associative algebras', J. Algebra 207 (1998), 491-510.

[5] A. van den Essen and V. Shpilrain, 'Some combinatorial questions about polynomial maps', J. Pure Appl. Algebra 119 (1997), 47-52.

[6] S.-J. Gong and J.-T. Yu, 'The linear coordinate preserving problem', Comm. Algebra 36 (2008), 1354-1364.

[7] S.-J. Gong and J.-T. Yu, 'Test elements, retracts and automorphic orbits', J. Algebra 320 (2008), 3062-3068.

[8] Z. Jelonek, 'A solution of the problem of van den Essen and Shpilrian', J. Pure Appl. Algebra 137 (1999), 49-55.

[9] Z. Jelonek, 'Test polynomials', J. Pure Appl. Algebra 147 (2000), 125-132.

[10] R. Lyndon and P. Schupp, Combinatorial Group Theory. Reprint of the 1977 edition, Classics in Mathematics (Springer, Berlin, 2001).

[11] J. H. McKay and S. S. S. Wang, 'An inversion formula for two polynomials in two variables', J. Pure Appl. Algebra 40 (1986), 245-257.

[12] A. A. Mikhalev and A. A. Zolotykh, 'The rank of an element of the free color Lie (p-) superalgebra', Dokl. Akad. Nauk 334 (1994), 690-693; English translation: Russian Acad. Sci. Dokl. Math. 49 (1994)(1), 189-193.

[13] V. Shpilrain and J.-T. Yu, 'Polynomial retracts and the Jacobian conjecture', Trans. Amer. Math. Soc. 352 (2000), 477-484. 
[14] V. Shpilrain and J.-T. Yu, 'Test polynomials, retracts, and the Jacobian conjecture', in: Affine Algebraic Geometry, Contemporary Mathematics, 369 (American Mathematical Society, Providence, RI, 2005), pp. 253-259.

[15] U. U. Umirbaev, 'On ranks of elements of free groups', Fundam. Prikl. Mat. 2 (1996), 313-315.

[16] Q. Yang, 'Retracts, test elements and automorphic orbit problem for polynomial algebras'. Master's Thesis, Jilin University, China, 2010.

[17] J.-T. Yu, 'Automorphic orbit problem for polynomial algebras', J. Algebra 319 (2008), 966-970.

YONG JIN, School of Mathematics, Jilin University,

130012 Changchun, PR China

e-mail: kingmeng@126.com

XIANKUN DU, School of Mathematics, Jilin University,

130012 Changchun, PR China

e-mail:duxk@jlu.edu.cn 